Article

\title{
Improving the Barrier Properties of Packaging Paper by Polyvinyl Alcohol Based Polymer Coating-Effect of the Base Paper and Nanoclay
}

\author{
Zhenghui Shen ${ }^{1}$, Araz Rajabi-Abhari ${ }^{1}\left(\mathbb{D}\right.$, Kyudeok Oh $^{2} \mathbb{D}$, Guihua Yang ${ }^{3}$, Hye Jung Youn ${ }^{1,2,3} \mathbb{D}$ \\ and Hak Lae Lee ${ }^{1,2,3, *}$ \\ 1 Program in Environmental Materials Science, Department of Agriculture, Forestry and Bioresources, \\ College of Agriculture and Life Sciences, Seoul National University, Seoul 08826, Korea; \\ zhshen@snu.ac.kr (Z.S.); araz61@snu.ac.kr (A.R.-A.); page94@snu.ac.kr (H.J.Y.) \\ 2 Research Institute of Agriculture and Life Sciences, Seoul National University, Seoul 08826, Korea; \\ ogd0310@snu.ac.kr \\ 3 State Key Laboratory of Biobased Material and Green Papermaking, Qilu University of Technology, \\ Shandong Academy of Sciences, Jinan 250353, China; ygh@qlu.edu.cn \\ * Correspondence: lhakl@snu.ac.kr
}

check for

updates

Citation: Shen, Z.; Rajabi-Abhari, A.; Oh, K.; Yang, G.; Youn, H.J.; Lee, H.L. Improving the Barrier Properties of Packaging Paper by Polyvinyl Alcohol Based Polymer Coating-Effect of the Base Paper and Nanoclay. Polymers 2021, 13, 1334 https://doi.org/10.3390/polym13081334

Academic Editor: Boxin Zhao

Received: 2 April 2021

Accepted: 16 April 2021

Published: 19 April 2021

Publisher's Note: MDPI stays neutral with regard to jurisdictional claims in published maps and institutional affiliations.

Copyright: (c) 2021 by the authors. Licensee MDPI, Basel, Switzerland. This article is an open access article distributed under the terms and conditions of the Creative Commons Attribution (CC BY) license (https:// creativecommons.org/licenses/by/ $4.0 /)$

\begin{abstract}
The poor barrier properties and hygroscopic nature of cellulosic paper impede the wide application of cellulosic paper as a packaging material. Herein, a polyvinyl alcohol (PVA)-based polymer coating was used to improve the barrier performance of paper through its good ability to form a film. Alkyl ketene dimer (AKD) was used to enhance the water resistance. The effect of the absorptive characteristics of the base paper on the barrier properties was explored, and it was shown that surface-sized base paper provides a better barrier performance than unsized base paper. Nanoclay (Cloisite $\mathrm{Na}^{+}$) was used in the coating formulation to further enhance the barrier performance. The results show that the coating of PVA/AKD/nanoclay dispersion noticeably improved the barrier performance of the paper. The water vapor transmission rate of the base paper was $533 \mathrm{~g} / \mathrm{m}^{2}$. day, and it decreased sharply to $1.3 \mathrm{~g} / \mathrm{m}^{2}$. day after the application of a double coating because of the complete coverage of the base paper by the PVA-based polymer coating. The coated paper had excellent water resistance owing to its high water contact angle of around $100^{\circ}$. The grease resistance and mechanical properties of the base paper also improved after coating. This work may provide inspiration for improving the barrier properties of packaging paper through the selection of a suitable base paper and coating formulation.
\end{abstract}

Keywords: packaging paper; barrier properties; polyvinyl alcohol; alkyl ketene dimer; nanoclay

\section{Introduction}

Packaging materials play important roles in many industries, such as the food industry and pharmaceutical industry, as well as in people's daily life. The basic function of packaging materials is to protect the contents from contaminants, leakage and damage in the production, storage, transportation and selling processes, and to provide safety, convenience and economic benefits [1]. Plastic, glass, metal and paper are the most commonly used packaging materials, among which plastic has been predominant in the global packaging market. Although plastic materials are light, flexible and strong, most of these petroleum-based products are nonrenewable and nonbiodegradable and have caused serious environmental issues [2]. A huge amount of plastic waste, including plastic packaging, is polluting the land and oceans and endangering ecosystems and the wildlife they contain. Paper packaging materials made from cellulosic fibers have the advantages of abundant availability, biodegradability, renewability and recyclability [3]. Furthermore, paper packaging materials are flexible, low cost and safe [4], and their good printability and ease of functionalization make them competitive in the packaging field. With rising 
awareness of environmental protection and sustainable development in society, paperbased packaging is attracting ever-increasing interest.

The barrier properties of packaging materials are critical for the protection of some products, such as moisture-sensitive foods and electronic devices. In this respect, cellulosic paper is not a perfect material for packaging owing to its poor barrier properties, which can mainly be ascribed to its porous three-dimensional structure. Because of the pores present both on the surface and inside the paper, molecules can diffuse through paper packaging rather easily and spoil the contents inside. Therefore, the porous nature prevents paper from fulfilling the protective function of a packaging material, thus limiting its application in the packaging sector. Additionally, the poor water resistance of paper, originating from the hydroxyl groups of cellulosic fibers, largely constrains its use as a packaging material in humid conditions. Upon contact with water, hygroscopic paper deteriorates and loses strength due to water absorption [5].

Intensive efforts have been made to improve the barrier properties of paper and paperboard products. Lavoine et al. [6] studied the effect of microfibrillated cellulose (MFC) on the barrier properties of paper and found that the air resistance of paper improved noticeably after a coating of MFC was applied. However, the grease resistance of the MFC-coated paper was not satisfactory. Mousavi et al. [7] coated cellulose nanofiber and carboxymethyl cellulose blends on paperboards and concluded that the coating improved the structure and barrier properties of the paper considerably. This coating also greatly improved the air resistance of paper, but the water vapor transmission rate (WVTR) and grease resistance of coated papers remained unsatisfactory. Hult et al. [8] attempted to enhance the barrier properties of paperboard by applying a lignin-based coating and found that the WVTR of the paperboard decreased from 840 to $240 \mathrm{~g} / \mathrm{m}^{2}$. day after the coating. Although improvements to the barrier properties of paper or paperboard through surface coating have been made in several studies, it is necessary to establish a better approach for coating in order to use paper products for the packaging of moisture-sensitive substances. In addition, the water resistance of paper, which is an important property for packaging applications, has been neglected in many studies. Hence, the fabrication of packaging paper with excellent barrier properties and water resistance remains an important objective.

To improve the barrier performance of porous paper, one effective method is to seal the pores on the paper's surfaces and thereby block the diffusion pathway of liquid/gas molecules through the paper. A common protocol is to coat polymers with good filmforming performance on the paper substrate and thus form a continuous and impermeable film. Polyvinyl alcohol (PVA) is widely used for its various advantages, including excellent film-forming ability and good barrier performance and mechanical properties. The filmforming ability of PVA-based coatings on paper surfaces improves the barrier performance because the continuous PVA film blocks the pores on the surface and hinders the diffusion of permeating molecules. However, a PVA coating tends to have poor water resistance owing to the hydrophilic and water-soluble nature of PVA. In this study, alkyl ketene dimer (AKD), which has been extensively used as a hydrophobizing agent in paper mills to enhance the water resistance of paper, was added to the PVA coating to improve the hydrophobicity of PVA-coated papers. The combined use of PVA and AKD to improve the barrier performance of paper was tested in our previous work [9]. Our results showed that the WVTR of paper decreased considerably, from 543 to $2 \mathrm{~g} / \mathrm{m}^{2}$.day, when we applied a PVA/AKD triple coating. However, the PVA/AKD coating has a disadvantage in terms of the cost-effectiveness of decreasing the WVTR. The addition of a nanofiller (e.g., nanoclay) has been considered an efficient way to enhance the barrier property of polymer-based coating materials. Several studies [10-12] have demonstrated the effectiveness of nanoclay in improving barrier performance through the tortuosity effect.

In the present study, PVA-compatible nanoclay $\left(\right.$ Cloisite $\mathrm{Na}^{+}$) was used as a nanofiller of a PVA-based polymer coating. By applying the PVA/AKD/nanoclay ternary coating material on the surface of paper, we aimed to prepare packaging paper with notably improved barrier performance and water resistance. Before coating the paper with this ternary 
dispersion, the influence of the base paper on improving the barrier performance was explored. Then, the effect of the PVA/AKD/nanoclay coating on the barrier performance of the paper was investigated to find cost-effective ways of fabricating packaging paper with superior barrier and water resistance properties.

\section{Materials and Methods}

\subsection{Materials}

Two types of base paper made of bleached eucalyptus kraft pulp with and without surface sizing were kindly provided by Moorim Paper Co. Ltd. (Jinju, Korea). The surface sizing was performed by applying $3 \mathrm{~g} / \mathrm{m}^{2}$ of oxidized starch to the surface of the dried web using a size press. The basic properties of these base papers are listed in Table 1. PVA (Polinol F17A, molecular weight $=75,000-80,000$, degree of hydrolysis $=98-99.5 \%$ ) was manufactured by OCI Company Ltd. (Seoul, Korea). AKD emulsion (HerconTM WI sizing agent) with a milky appearance and a solids content of $19.62 \%$ was supplied by Solenis Korea Ltd. (Gimcheon, Korea). Acridine orange (AO) was purchased from Samchun Chemical Co., Ltd. (Seoul, Korea). Nanoclay $\left(\right.$ Cloisite $\mathrm{Na}^{+}$) was obtained from Southern Clay Products Inc. (Gonzales, TX, USA). EpoFix resin (Struers, Cleveland, OH, USA) was used for embedding the coated samples. All of the materials and chemicals were used as received without any further purification or modification.

Table 1. Comparison of the properties of the two base papers.

\begin{tabular}{ccc}
\hline Base Paper & Surface-Unsized & Surface-Sized \\
\hline Basis weight $\left(\mathrm{g} / \mathrm{m}^{2}\right)$ & 84 & 81 \\
Thickness $(\mu \mathrm{m})$ & 99.25 & 97.12 \\
Bulk $\left(\mathrm{cm}^{3} / \mathrm{g}\right)$ & 1.18 & 1.20 \\
Density $\left(\mathrm{g} / \mathrm{cm}^{3}\right)$ & 0.85 & 0.83 \\
PPS $^{1}$ roughness $(\mu \mathrm{m})$ & 4.53 & 5.46 \\
Brightness $\left.(\%)^{2}\right)$ & 92.81 \\
WVTR $^{2}\left(\mathrm{~g} / \mathrm{m}^{2}\right.$.day $)$ & 543.11 & 533.01 \\
\hline
\end{tabular}

${ }^{1}$ PPS $=$ Parker Print-Surf, ${ }^{2}$ WVTR $=$ water vapor transmission rate.

\subsection{Methods}

\subsubsection{Preparation of Coating Dispersions}

The coating formulations are shown in Table 2. A solution with 8\% PVA was prepared by dissolving the required amount of PVA powder in deionized water at $90{ }^{\circ} \mathrm{C}$ for $1 \mathrm{~h}$, during which the dispersion was mixed with a mechanical stirrer (WiseStir, Daihan Scientific Co., Ltd., Wonju, Korea) at a speed of $800 \mathrm{rpm}$. To the PVA solution, the AKD emulsion was added and the mixture was mechanically mixed at $900 \mathrm{rpm}$ for $1 \mathrm{~h}$. Then, nanoclay was added and sonication with a frequency of $28 \mathrm{kHZ}$ was conducted for $2 \mathrm{~h}$ using an ultrasound cleaner (SH-2300 Model, Saehan Ultrasonic, Seoul, Korea). Three levels of nanoclay dosage (i.e., $3 \%, 5 \%$ and $10 \%$ ) were applied in the coating formulations. The prepared PVA/AKD/nanoclay dispersions were mechanically mixed at $1000 \mathrm{rpm}$ at room temperature for $1 \mathrm{~h}$ before coating.

Table 2. Coating formulations.

\begin{tabular}{ccccc}
\hline Coating & \#1 & \#2 & \#3 & \#4 \\
\hline PVA $\left(\mathrm{pph}^{1}\right)$ & 100 & 100 & 100 & 100 \\
AKD $\left(\mathrm{pph}^{1}\right)$ & 15 & 15 & 15 & 15 \\
Nanoclay $(\mathrm{pph})$ & 0 & 3 & 5 & 10 \\
\hline
\end{tabular}

${ }^{1}$ Component amounts are reported as parts per hundred (pph; by weight) based on 100 parts of PVA. PVA = polyvinyl alcohol; $\mathrm{AKD}=$ alkyl ketene dimer. 


\subsubsection{Coating Process}

The coating was carried out with an autobar coater (GBC-A4, GIST Co., Daejeon, Korea). The coating speed was $70 \mathrm{~mm} / \mathrm{s}$. Coated papers were dried in a hot air dryer (HB-502P0, Hanbaek Scientific Co., Bucheon, Korea) for $3 \mathrm{~min}$ and conditioned following TAPPI standard T $402 \mathrm{sp}-13$ (TAPPI 2013). The properties of the coated papers were then determined.

\subsubsection{Confocal Laser Scanning Microscope (CLSM) Imaging}

The penetration of the coating materials into the base papers was investigated using a CLSM (SP8 X, Leica, Wetzlar, Germany). To this end, PVA was stained with AO in darkness for $6 \mathrm{~h}$ and then coated on the surfaces of the base papers in darkness. The coated papers were embedded in epoxy resin, followed by the curing (room temperature) and polishing (LaboPo-5 polisher, Struers, Cleveland, OH, USA) of the prepared resin blocks. The cross-sections of coated papers were examined under the CLSM and the acquired CLSM images were processed using software Leica Application Suite X (LAS X, Version 3.5.1, Leica, Wetzlar, Germany).

\subsubsection{Surface Morphologies of Papers}

The surface morphologies of the papers were recorded by field-emission scanning electron microscopy (FE-SEM, Supra 55VP, Carl Zeiss, Oberkochen, Germany) with an acceleration voltage of $2 \mathrm{kV}$. The platinum coating of samples was performed before FE-SEM imaging using a sputter coater (EM ACE200, Leica, Vienna, Austria).

\subsubsection{Fourier-Transform Infrared (FT-IR) Spectroscopy}

FT-IR spectra were recorded using a Nicolet 6700 spectrometer equipped with an attenuated total reflection accessory (Thermo Scientific, Waltham, MA, USA). The spectra were acquired with 32 scans in the range of $4000-1000 \mathrm{~cm}^{-1}$ at a resolution of $8 \mathrm{~cm}^{-1}$.

\subsubsection{Measurements of the Water Contact Angle (WCA)}

The WCAs of the papers were determined using a drop shape analyzer (DSA100, Krüss GmbH, Hamburg, Germany). WCAs were measured for $60 \mathrm{~s}$ and the measurement interval was $1 \mathrm{~s}$. Deionized water was used as the testing liquid and the volume of the water droplets was $5 \mu \mathrm{L}$. Six measurements were carried out for each sample and the average values were then calculated and reported.

\subsubsection{Measurements of the Cobb60 Value}

The Cobb60 values of the papers were measured at $23{ }^{\circ} \mathrm{C}$ and $50 \%$ relative humidity (RH) according to TAPPI standard T $441 \mathrm{om}-13$ (TAPPI 2013). The average of at least three measurements was calculated.

\subsubsection{Measurements of the WVTR and Grease Resistance}

The WVTRs of the papers were measured at $23{ }^{\circ} \mathrm{C}$ and $50 \% \mathrm{RH}$ following TAPPI standard T $448 \mathrm{om}-17$ (TAPPI 2017). Grease resistance measurements of the papers were performed according to TAPPI standard T $559 \mathrm{~cm}-12$ (TAPPI 2012). At least three measurements were made for each paper and the average values were reported.

\subsubsection{Mechanical Properties of Papers}

The tensile properties of the papers were examined using a universal testing machine (Instron 5943, Instron Corp., Norwood, MA, USA) equipped with a 500-N load cell. Specimens were $15 \mathrm{~mm}$ in width and $100 \mathrm{~mm}$ in length and the tensile testing was carried out at $23{ }^{\circ} \mathrm{C}$ and $50 \% \mathrm{RH}$. Average values of the elongation at break and tensile strength were calculated and reported. 


\section{Results and Discussion}

\subsection{Comparison of the Properties of the Two Base Papers}

The two base papers were similar in terms of several properties (Table 1), including the basis weight, thickness, bulk, density, roughness, brightness and WVTR. However, the liquid absorptive characteristics (Figure 1) differed greatly. The WCA results (Figure 1a) show that the two base papers had similar WCAs at the initial moment of testing. As time advanced, the WCA of the surface-sized base paper largely remained constant. In contrast, the WCA of the unsized base paper decreased sharply with testing time, finally reaching $11^{\circ}$, which is a value much lower than the WCA of the surface-sized base paper. The unsized base paper had poor water resistance due to the hydrophilic and porous nature of paper [13], which will allow easier absorption and penetration of water-borne coatings into the paper. The result of Cobb60 testing (Figure 1b) confirmed the different liquid absorptive performances of the two base papers. This difference may lead to different absorption or penetration of coating materials into the different base papers, which would consequently affect the barrier performance of PVA-based coated papers even for the same coat weight.
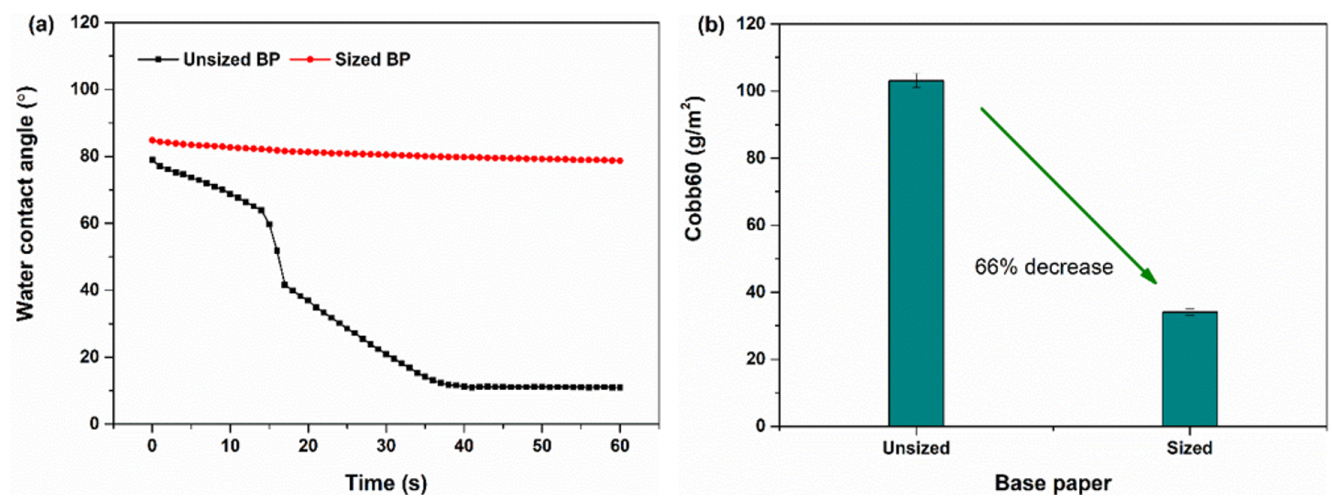

Figure 1. Characteristics of the two base papers: (a) WCAs and (b) Cobb60 values. BP = base paper.

\subsection{Effect of the Base Paper on the WVTR of Coated Papers}

AO, a green fluorescent dye, has been used to stain fibers [14], PVA [15] and starch [16]. Hence, to investigate the penetration of PVA into the base papers, the PVA was stained with $\mathrm{AO}$ before the coating process. The cross-sections of the coated papers were then analyzed under the CLSM, and the intensity and distribution of the emitted fluorescence were determined and compared between the coated papers (Figure 2).

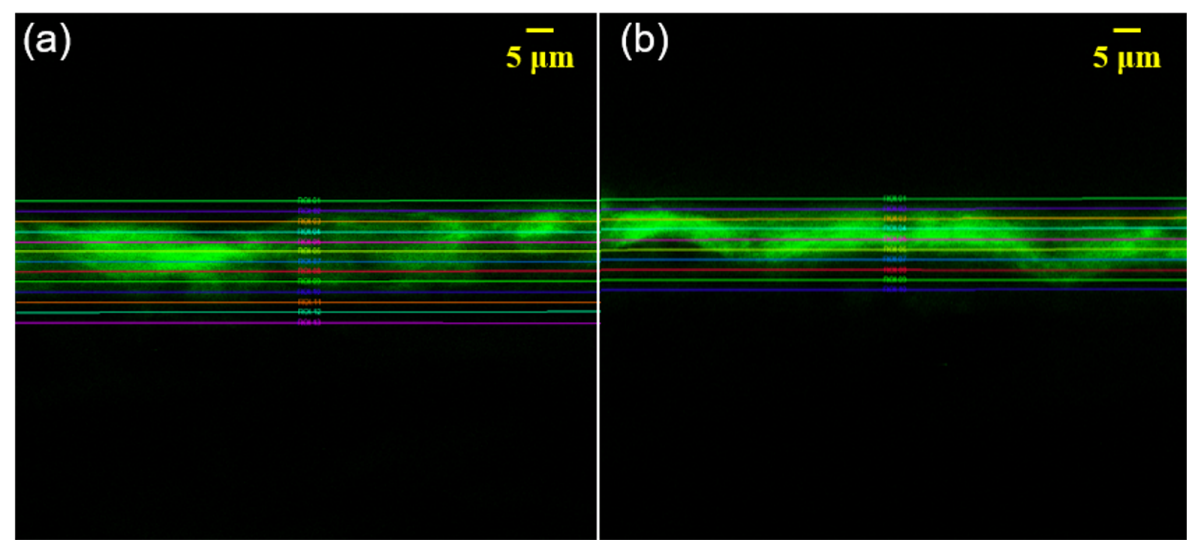

Figure 2. CLSM images of AO-stained PVA-coated papers: coatings on (a) the unsized base paper and (b) surface-sized base paper.

When AO-stained PVA was coated on the surface of the unsized base paper, the overall intensity of the fluorescence (Figure 2a) was not as strong as that on the surface-sized base 
paper (Figure 2b). Additionally, Figure 2a shows a deeper penetration of the coating into the unsized base paper, which agreed well with the results presented in Figure 1. When surface-sized paper was used as the substrate for coating, less penetration by the coating material was observed. When the same amount of coating dispersion was applied on the surface of paper, more penetration by the coating meant that less of the coating remained on the surface. This would impede film formation on the surface, and this unfavorably affects the barrier performance of polymer-coated papers.

The WVTR values of the PVA-coated papers were determined to confirm the above inferences. Figure 3 shows that a PVA coating of either the unsized or surface-sized base paper reduced the WVTR substantially, relative to the base paper before PVA coating, indicating that the PVA coating is highly effective in improving the barrier performance of paper. As expected, the WVTR of the coated paper was even lower when the surface-sized base paper was used as the coating substrate, which could be attributed to the weaker penetration by coating materials and improved PVA film formation on the paper surface. Less penetration of the PVA coating into the base paper means not only an improvement in barrier performance but also a reduction in the cost of the PVA required.

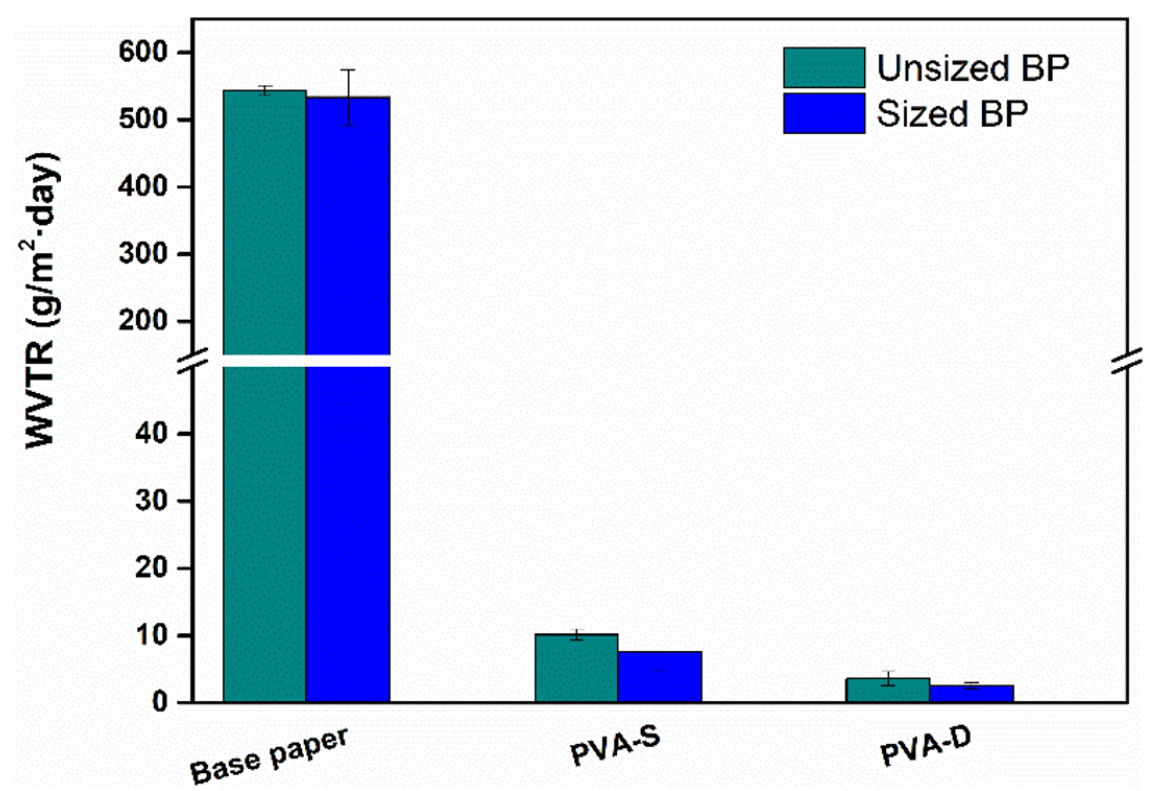

Figure 3. WVTR of papers. S: single coating; D: double coating; BP: base paper.

\subsection{Analysis of the Surface Coverage and Chemical Interactions}

The surface-sized base paper was used in the following experiments, considering its advantage in preventing the penetration of the coating material. The surface morphologies of the base paper, PVA/AKD-coated papers and PVA/AKD/nanoclay-coated papers are shown in Figure 4, and the coat weights are presented in Table 3. The porous nature of the base paper surface, shown in Figure 4a, would allow easy penetration of fluid molecules through the typical porous structure of paper. Therefore, blocking the pores on the surface of the base paper is a simple and effective way to improve the barrier properties. A single coating of PVA/AKD (Figure $4 \mathrm{~b}$ ) failed to fully cover the surface of the base paper, and some pores remained on the surface. However, with the application of a double coating of $\mathrm{PVA} / \mathrm{AKD}$, the coating coverage of the paper surface improved and a complete sealing of all pores by the PVA/AKD film was achieved (Figure 4d). This indicates that a double coating of PVA/AKD would greatly improve the barrier performance as a well-formed film on the paper surface would prevent liquid/gas permeation. The same phenomenon was observed for the PVA/AKD/nanoclay-coated papers (Figure 4c,e), i.e., a single coating failed to seal all pores on the surface of the base paper while a double coating fully covered the surface pores and gave a satisfactory coating coverage. Considering that a continuous 
solid film on the surface of the paper was the key to improving barrier performance, the properties of the double-coated papers were investigated in the followed sections.

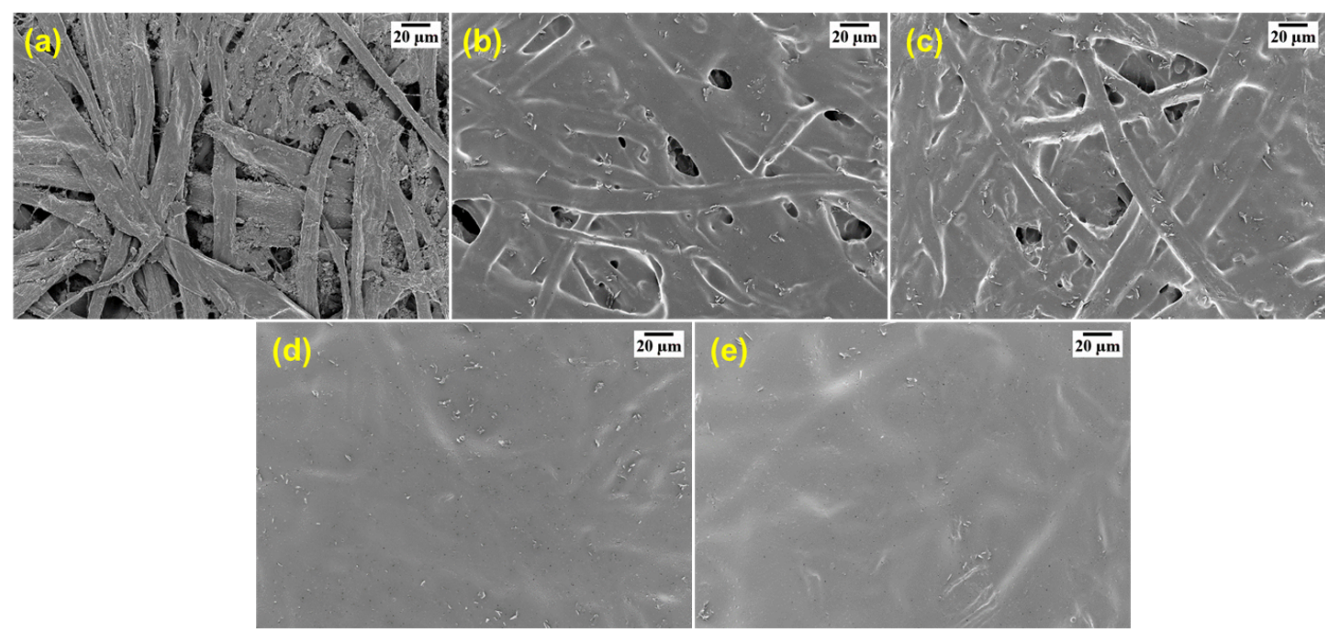

Figure 4. FE-SEM images of (a) the base paper, (b) PVA/AKD-S coated paper, (c) PVA/AKD/5\% nanoclay-S coated paper, (d) PVA/AKD-D coated paper, and (e) PVA/AKD/5\% nanoclay-D coated paper. S: single coating; D: double coating.

Table 3. Coat weights of the papers.

\begin{tabular}{|c|c|c|}
\hline Formulation & Single Coating & Double Coating \\
\hline PVA/AKD & $6.51 \pm 0.17^{1}$ & $11.79 \pm 0.16$ \\
\hline PVA/AKD/3\% nanoclay & $6.55 \pm 0.08$ & $12.28 \pm 0.17$ \\
\hline PVA/AKD/5\% nanoclay & $6.52 \pm 0.16$ & $12.08 \pm 0.16$ \\
\hline PVA/AKD/10\% nanoclay & $6.62 \pm 0.16$ & $12.46 \pm 0.21$ \\
\hline
\end{tabular}

${ }^{1}$ The coat weight is reported as $\mathrm{g} / \mathrm{m}^{2}$.

The FT-IR spectra of the coated papers are shown in Figure 5. The broad band centered at $3335 \mathrm{~cm}^{-1}$ in the spectrum of the base paper is attributed to the stretching vibration of $-\mathrm{OH}$ (hydroxyl) groups [17], and these hydroxyl groups of cellulose contribute to the hygroscopic nature of paper-based materials. Similarly, the spectrum of PVA has a strong band at $3270 \mathrm{~cm}^{-1}$, which can be assigned to the $\mathrm{O}-\mathrm{H}$ stretching of the abundant hydroxyl groups in PVA films [18]. Therefore, although the formation of PVA film on the surface of paper can block the pores and improve the barrier performance, it also increases the hydrophilicity of paper because of the abundant hydroxyl groups of PVA. In the spectrum of AKD, the band at $1847 \mathrm{~cm}^{-1}$ represents the typical lactone ring of AKD molecules [19], and the bands at 1720 and $1647 \mathrm{~cm}^{-1}$ represent $C=O$ and $C=C$ stretching vibrations of $A K D$, respectively. A new band appeared at $1703 \mathrm{~cm}^{-1}$ in the spectrum of the PVA/AKD-coated paper, which indicated the formation of a $\beta$-ketoester [20]. During the drying process, AKD reacted with the hydroxyl groups of the PVA, which would enhance the hydrophobicity of the PVA-coated paper.

The spectrum of nanoclay has a strong band at $3623 \mathrm{~cm}^{-1}$, which can be assigned to the $\mathrm{O}-\mathrm{H}$ stretching of $\mathrm{Al}-\mathrm{OH}$ and $\mathrm{Si}-\mathrm{OH}$ structures of nanoclay [21]. Interestingly, this band was not visible in the spectrum of the PVA/AKD/nanoclay-coated paper, possibly because nanoclay was embedded in the PVA/AKD matrix and could not be detected. Meanwhile, the band at $1703 \mathrm{~cm}^{-1}$ ( $\beta$-ketoester) was also visible in the spectrum of the $\mathrm{PVA} / \mathrm{AKD} /$ nanoclay-coated paper. This again suggests that chemical reactions occurred between AKD and the hydroxyl groups of PVA, which may have resulted in the improved water resistance of the paper. 


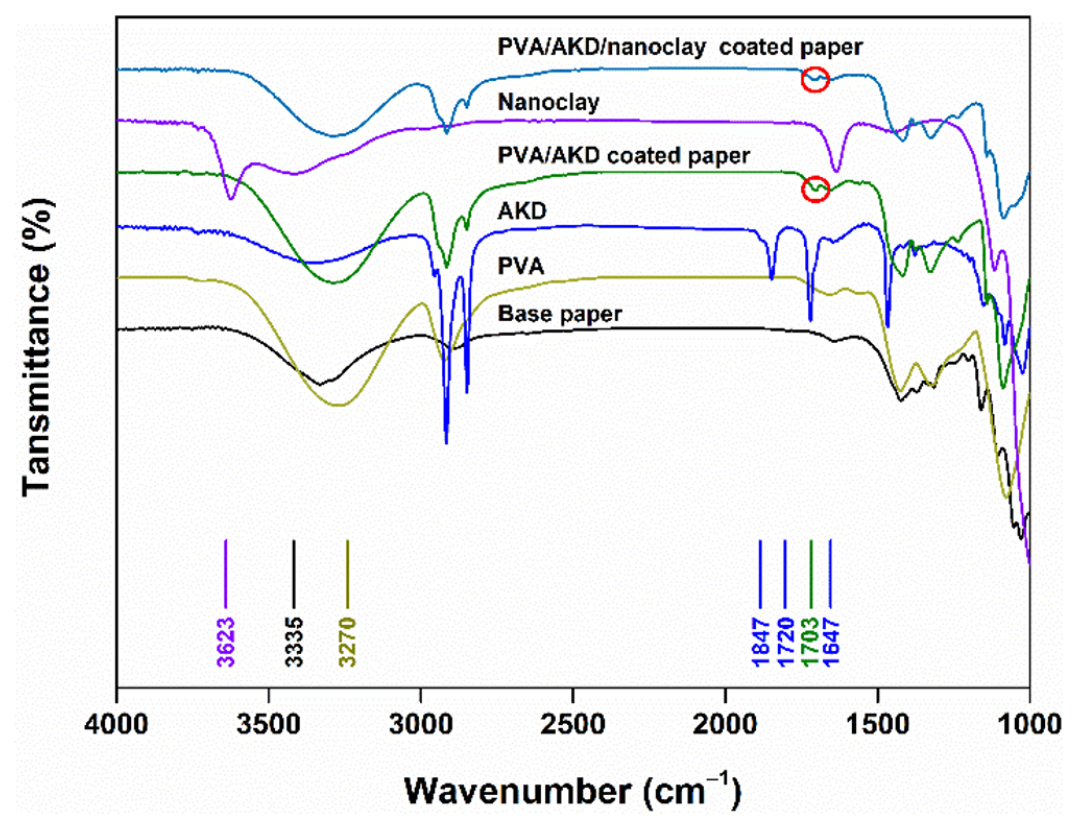

Figure 5. FT-IR spectra of coated papers and the coating components.

\subsection{Water Resistance and Barrier Performance of Coated Papers}

The WCAs of the coated papers were determined and compared with the WCA of the base paper (Figure 6). Although the base paper was surface-sized, the WCA slightly decreased with time. When the AKD was incorporated into the coating formulation, the WCAs of the coated papers increased, showing the effectiveness of AKD in increasing the water repellency of paper. The hydrophobizing agent AKD can react with the hydroxyl group of PVA and cellulose to form ester bonds [22], and thus decrease the hydrophilicity of the coated paper, which was confirmed in the FT-IR analysis (Figure 5). The addition of nanoclay slightly increased the WCA of the coated papers, and it appeared that the change in surface morphology of the coated papers contributed to this phenomenon [23]. Importantly, the WCAs of the coated papers remained constant during the testing, indicating good water resistance was achieved, which would enhance the applicability of the resulting coated papers in humid or even wet conditions.

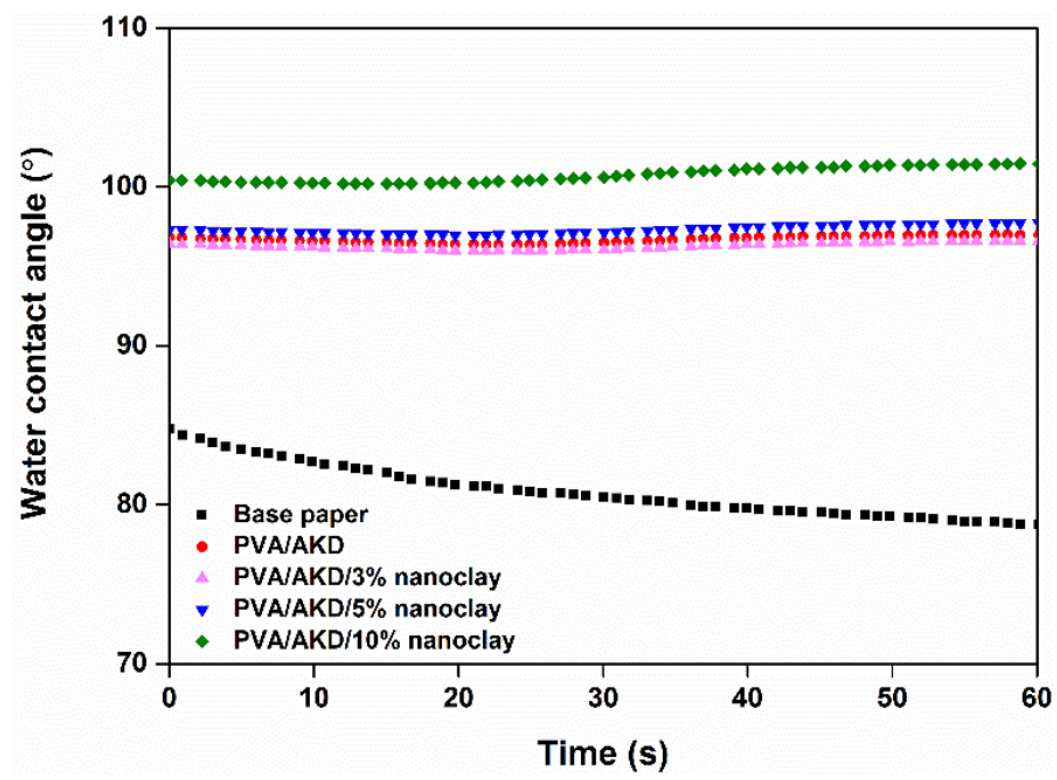

Figure 6. WCAs of the base paper and coated papers. 
A Cobb value represents the amount of water absorbed by paper during a specific period, such as $60 \mathrm{~s}$ in this study. The value is affected by various characteristics of the paper, such as the porosity and sizing. A smaller $\mathrm{Cobb}$ value indicates better water resistance of the coated paper. Figure 7 shows that the Cobb60 values of the coated papers were much lower than that of the base paper because the PVA coating layers decreased both the number and the size of the pores. Additionally, Hu et al. [24] showed that eliminating the pores in paper was an effective method of improving water resistance. This was consistent with the present study's finding that sizing remarkably reduced the water absorption of paper, as shown in Figure 1b. These results give insight into controlling water resistance by altering the characteristics of paper.



Figure 7. Cobb60 values of the base paper and coated papers.

The WVTR is an important barrier property of packaging materials for moisturesensitive products. The WVTRs of the base paper and coated papers were examined (Figure 8). Compared with the base paper, the coated papers had much lower WVTRs. PVA formed a dense polymer film on the paper surface and decreased the number of pores. A higher coat weight further improved the barrier performance of the papers (Figure 3) because it provided more complete coverage of the paper surface. There was no big difference in the absolute values of WVTR for the coated papers because the coating of PVA/AKD had already decreased the WVTR of paper to a low level $\left(1.90 \mathrm{~g} / \mathrm{m}^{2}\right.$.day). The use of $3 \%$ nanoclay in the coating formulation further decreased the WVTR value to $1.47 \mathrm{~g} / \mathrm{m}^{2}$.day, which was $22.6 \%$ lower than that of PVA/AKD coated paper. The use of $5 \%$ nanoclay decreased the WVTR value to $1.26 \mathrm{~g} / \mathrm{m}^{2}$.day, which was $33.7 \%$ lower than that of PVA/AKD coated paper. These improvements can be attributed to the tortuosity effect of this platy nano-sized material [25], i.e., the presence of nanoclay in a PVA-based coating prevents the diffusion of permeating molecules by making the molecules follow a more tortuous pathway. However, when $10 \%$ nanoclay was used, the WVTR of the coated paper increased. It is known that the tortuosity effect is dependent on the dispersion of the nanoclay in the coating materials. When an excessive amount of nanoclay is present in the polymer matrix, an agglomeration of nanoclay generates interfacial voids at the particle/polymer matrix interface and forms a preferential pathway for the diffusion of water vapor molecules [26]. In the present study, the addition of $5 \%$ nanoclay gave the optimal water vapor resistance, suggesting that neither insufficient nor excess use of nanoclay provides an ideal tortuosity effect owing to the lack of nanoplates or agglomeration of nanoclay in the diffusion pathway of water vapor [27]. Thus, selecting suitable nanofillers and optimizing their dosage is critical for obtaining a coated paper with high barrier performance. 
In our previous work [9], the barrier properties of papers were enhanced by applying different layers of PVA/AKD coatings on the surface of a base paper. The lowest WVTR value was around $2 \mathrm{~g} / \mathrm{m}^{2}$.day when the triple coating of PVA/AKD $\left(11 \mathrm{~g} / \mathrm{m}^{2}\right.$ coat weight $)$ was applied on the surface of the base paper. However, it was difficult to further decrease the WVTR by coating more PVA/AKD on the surface of the triple-coated paper, i.e., quadruple coating $\left(15 \mathrm{~g} / \mathrm{m}^{2}\right.$ coat weight). Interestingly, a lower WVTR value was achieved by double coating ( $12 \mathrm{~g} / \mathrm{m}^{2}$ coat weight) of PVA/AKD $/ 5 \%$ nanoclay on the surface of the paper, which again confirmed the effectiveness of using nanoclay.

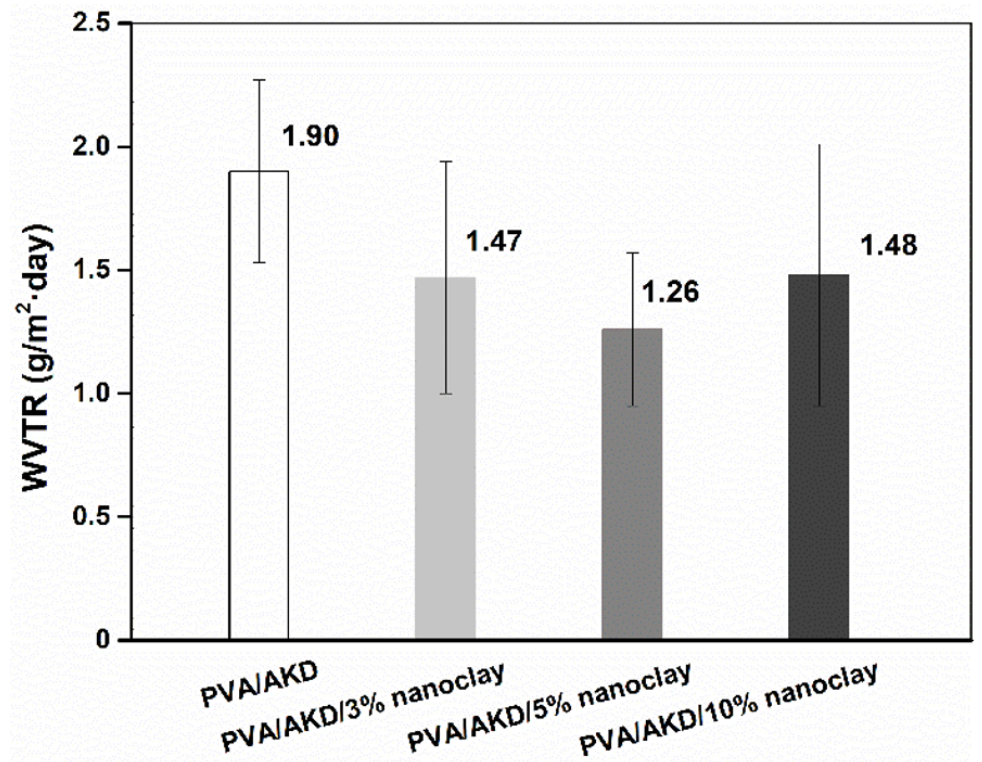

Figure 8. WVTR of the base paper and coated papers.

Grease resistance is another major factor affecting the barrier performance of packaging papers, especially for the packaging of oily foods and certain machinery accessories. The grease resistance of the papers was determined according to TAPPI standard T 559 $\mathrm{cm}-12$ (Figure 9), where a kit value ranging from 0 to 12 is used to grade the repellency of paper to grease and oil. A higher kit value indicates a greater grease resistance of a paper. The results showed the base paper had a kit value of 0 , indicating that it has no grease resistance. In contrast, all coated papers had a kit value of 12 , showing an extraordinary improvement in grease resistance. Two factors contributed to the satisfactory grease resistance of these coated papers. First, PVA itself has strong grease resistance [28]. This is seen from the kit value of the PVA-coated paper (red dot in Figure 9). Second, the sealing of the pores on the surface of base paper prevents the penetration of grease [29]. A PVA coating improves the resistance against grease by forming a continuous film on paper surfaces with high oil resistance. One thing to point out is that the same kit values were always obtained in the grease resistance test of each sample. For example, in the grease resistance test of the base paper, a kit value of zero was obtained in each replicate measurement, suggesting the poor grease resistance of the sample and the good repeatability of the experiment. Therefore, the error bar is not visible in Figure 9.

\subsection{Strength Properties of Coated Papers}

The physical properties of papers play an important role in the converting process and end uses of the papers. For example, the tensile strength and extensibility of papers are indicative of the potential resistance to the web break of papers in a printing process or any other web-fed converting process [30]. In the case of sack paper, tensile strength and elongation affect how strong this packaging material can be and how much load it can carry. The elongation at break and tensile strength of the papers examined in this study are depicted in Figure 10. The base paper had an elongation at break of $2.2 \%$ and 
tensile strength of $3.5 \mathrm{kN} / \mathrm{m}$, which were much lower than the values for the coated papers. Specifically, the elongation at break of all coated papers was at least $32 \%$ higher than that of the base paper, and the tensile strength of coated papers was $23-25 \%$ higher than that of the base paper. This indicates that the coated paper can withstand a stronger stretching or deformation without breaking compared to the base paper. The tensile strength improved after PVA-based coating because PVA has a good reinforcing effect on the tensile strength of paper [31,32]. When nanoclay was used in the coating formulation, the mechanical properties of the coated papers remained similar. Thus, a conclusion can be made that the improvement in the elongation and tensile strength was mainly because of the PVA/AKD films. In other words, the use of nanoclay had little influence on the mechanical properties of coated papers. Our previous work showed that the coating of PVA/AKD improved water and grease resistance, and the mechanical properties [9].

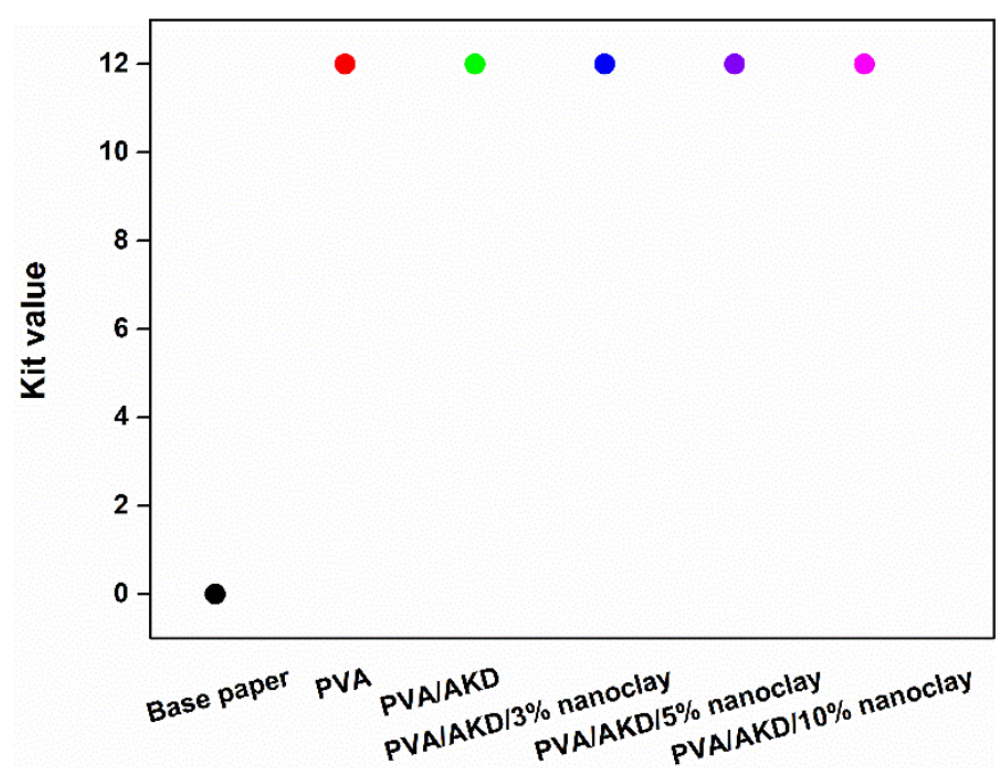

Figure 9. Kit values of the base paper and coated papers in grease resistance testing.

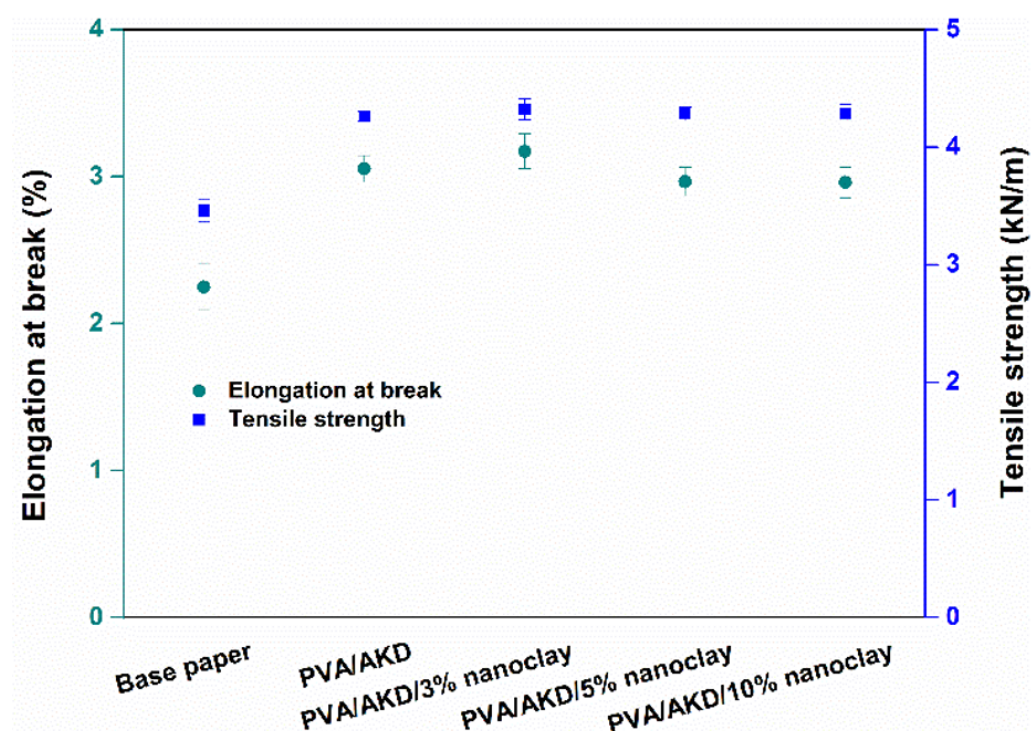

Figure 10. Elongations at break and tensile strengths of the base paper and coated papers.

\section{Conclusions}

In this work, the barrier performance and water resistance of cellulosic paper were enhanced by coating a PVA/AKD/nanoclay dispersion on the surface of paper. The 
effect of the absorptive characteristics of the base papers on the improvement in barrier performance was investigated by CLSM analysis. The results indicated that the use of surface-sized base paper contributed to a higher barrier performance owing to the impeded penetration of coatings and thus a favored film formation on the paper surface. The use of AKD in the coating formulation improved the water resistance of the papers, and the coated papers had WCAs of around $100^{\circ}$. The addition of $5 \%$ nanoclay in the coating formulation further enhanced the barrier performance of cellulosic paper owing to its tortuosity effect on the diffusion pathway, and the lowest WVTR value of a coated paper was $1.3 \mathrm{~g} / \mathrm{m}^{2}$.day when a double coating of PVA/AKD $/ 5 \%$ nanoclay dispersion was applied. The water and grease resistance of the coated papers improved notably, relative to those of the base paper. In summary, the PVA/AKD/nanoclay-coated papers exhibited hydrophobicity, excellent barrier performance and improved strength properties, which would expand the applications of cellulosic paper in the packaging industry.

Author Contributions: Conceptualization: Z.S.; methodology: Z.S.; investigation: Z.S.; writingoriginal draft: Z.S., A.R.-A. and K.O.; writing—review and editing: G.Y., H.J.Y., H.L.L.; funding acquisition, project administration, and supervision: H.L.L. All authors have read and agreed to the published version of the manuscript.

Funding: This research was funded by Paper Science Lab, Seoul National University. Author Zhenghui Shen acknowledges the support from China Scholarship Council (CSC, grant No. 201708120051).

Institutional Review Board Statement: Not applicable.

Informed Consent Statement: Not applicable.

Data Availability Statement: The data presented in this study are available on request from the corresponding author.

Conflicts of Interest: The authors declare no conflict of interest.

\section{References}

1. Domnica, D. Review concerning the functions of packaging. Land Forces Acad. Rev. 2010, 15, 44-48.

2. Johansson, C.; Bras, J.; Mondragon, I.; Nechita, P.; Plackett, D.; Simon, P.; Svetec, D.G.; Virtanen, S.; Baschetti, M.G.; Breen, C. Renewable fibers and bio-based materials for packaging applications-A review of recent developments. BioResources 2012, 7, 2506-2552. [CrossRef]

3. Herrera, M.A.; Mathew, A.P.; Oksman, K. Barrier and mechanical properties of plasticized and cross-linked nanocellulose coatings for paper packaging applications. Cellulose 2017, 24, 3969-3980. [CrossRef]

4. Tobjork, D.; Osterbacka, R. Paper electronics. Adv. Mater. 2011, 23, 1935-1961. [CrossRef]

5. Alava, M.; Niskanen, K. The physics of paper. Rep. Prog. Phys. 2006, 69, 669. [CrossRef]

6. Lavoine, N.; Desloges, I.; Khelifi, B.; Bras, J. Impact of different coating processes of microfibrillated cellulose on the mechanical and barrier properties of paper. J. Mater. Sci. 2014, 49, 2879-2893. [CrossRef]

7. Mazhari Mousavi, S.M.; Afra, E.; Tajvidi, M.; Bousfield, D.W.; Dehghani-Firouzabadi, M. Cellulose nanofiber/carboxymethyl cellulose blends as an efficient coating to improve the structure and barrier properties of paperboard. Cellulose 2017, 24, 3001-3014. [CrossRef]

8. Hult, E.-L.; Ropponen, J.; Poppius-Levlin, K.; Ohra-Aho, T.; Tamminen, T. Enhancing the barrier properties of paper board by a novel lignin coating. Ind. Crops Prod. 2013, 50, 694-700. [CrossRef]

9. Shen, Z.; Kwon, S.; Oh, K.; Abhari, A.R.; Lee, H.L. Facile fabrication of hydrophobic cellulosic paper with good barrier properties via PVA/AKD dispersion coating. Nord. Pulp Pap. Res. J. 2019, 34, 516-524. [CrossRef]

10. Tan, B.; Thomas, N.L. Tortuosity model to predict the combined effects of crystallinity and nano-sized clay mineral on the water vapour barrier properties of polylactic acid. Appl. Clay Sci. 2017, 141, 46-54. [CrossRef]

11. Mirmehdi, S.; Hein, P.R.G.; de Luca Sarantópoulos, C.I.G.; Dias, M.V.; Tonoli, G.H.D. Cellulose nanofibrils/nanoclay hybrid composite as a paper coating: Effects of spray time, nanoclay content and corona discharge on barrier and mechanical properties of the coated papers. Food Packag. Shelf Life 2018, 15, 87-94. [CrossRef]

12. Chang, J.-H. Comparative analysis of properties of PVA composites with various nanofillers: Pristine clay, organoclay, and functionalized graphene. Nanomaterials 2019, 9, 323. [CrossRef]

13. Mokhothu, T.H.; John, M.J. Review on hygroscopic aging of cellulose fibres and their biocomposites. Carbohydr. Polym. 2015, 131, 337-354. [CrossRef]

14. Ozaki, Y. Application of confocal laser scanning microscopy (CLSM) for observing adhesives in paper. J. Adhes. Sci. Technol. 2011, 25, 723-741. [CrossRef] 
15. Park, H.; Park, S.Y.; Yook, S.; Kim, T.-Y.; Youn, H.J. Impregnation of paper with cellulose nanofibrils and polyvinyl alcohol to enhance durability. Nord. Pulp Pap. Res. J. 2020, 35, 106-114. [CrossRef]

16. Seo, M.S.; Youn, H.J.; Lee, H.L. Penetration control of surface sizing starch using cationic PAM and its effect on the bending stiffness of paper. BioResources 2020, 15, 5489-5502. [CrossRef]

17. Peresin, M.S.; Habibi, Y.; Zoppe, J.O.; Pawlak, J.J.; Rojas, O.J. Nanofiber composites of polyvinyl alcohol and cellulose nanocrystals: Manufacture and characterization. Biomacromolecules 2010, 11, 674-681. [CrossRef]

18. Gohil, J.M.; Ray, P. Polyvinyl alcohol as the barrier layer in thin film composite nanofiltration membranes: Preparation, characterization, and performance evaluation. J. Colloid Interface Sci. 2009, 338, 121-127. [CrossRef]

19. Song, X.; Chen, F.; Liu, F. Preparation and characterization of alkyl ketene dimer (AKD) modified cellulose composite membrane. Carbohydr. Polym. 2012, 88, 417-421. [CrossRef]

20. Song, X.; Chen, F.; Liu, F. Study on the reaction of alkyl ketene dimer (AKD) and cellulose fiber. BioResources $2012,7,652-662$.

21. Mallakpour, S.; Dinari, M. Biomodification of cloisite $\mathrm{Na}^{+}$with L-methionine amino acid and preparation of poly(vinyl alcohol)/organoclay nanocomposite films. J. Appl. Polym. Sci. 2012, 124, 4322-4330. [CrossRef]

22. Lee, K.H.; Youn, H.J.; Lee, H.L. UV/Vis spectrometry-based analysis of alkyl ketene dimer (AKD) retention to solve the waxy spot problem in the papermaking process. ACS Omega 2020, 5, 11227-11234. [CrossRef] [PubMed]

23. Oliver, J.; Huh, C.; Mason, S. An experimental study of some effects of solid surface roughness on wetting. Colloids Surf. 1980, 1, 79-104. [CrossRef]

24. Hu, Z.; Zen, X.; Gong, J.; Deng, Y. Water resistance improvement of paper by superhydrophobic modification with microsized $\mathrm{CaCO}_{3}$ and fatty acid coating. Colloids Surf. A Physicochem. Eng. Asp. 2009, 351, 65-70. [CrossRef]

25. Wolf, C.; Angellier-Coussy, H.; Gontard, N.; Doghieri, F.; Guillard, V. How the shape of fillers affects the barrier properties of polymer/non-porous particles nanocomposites: A review. J. Membr. Sci. 2018, 556, 393-418. [CrossRef]

26. Ismail, A.; Rahim, N.; Mustafa, A.; Matsuura, T.; Ng, B.; Abdullah, S.; Hashemifard, S. Gas separation performance of polyethersulfone/multi-walled carbon nanotubes mixed matrix membranes. Sep. Purif. Technol. 2011, 80, 20-31. [CrossRef]

27. Nguyen, Q.T.; Baird, D.G. Preparation of polymer-clay nanocomposites and their properties. Adv. Polym. Technol. 2006, 25, 270-285. [CrossRef]

28. Spagnol, C.; Fragal, E.H.; Witt, M.A.; Follmann, H.D.M.; Silva, R.; Rubira, A.F. Mechanically improved polyvinyl alcoholcomposite films using modified cellulose nanowhiskers as nano-reinforcement. Carbohydr. Polym. 2018, 191, 25-34. [CrossRef]

29. Aulin, C.; Gällstedt, M.; Lindström, T. Oxygen and oil barrier properties of microfibrillated cellulose films and coatings. Cellulose 2010, 17, 559-574. [CrossRef]

30. Pratima, B. Paper and Its Properties. In Biermann's Handbook of Pulp and Paper, 3rd ed.; Pratima, B., Ed.; Elsevier: Amsterdam, The Netherlands, 2018; pp. 35-63.

31. Awada, H.; Bouatmane, M.; Daneault, C. High strength paper production based on esterification of thermomechanical pulp fibers in the presence of poly (vinyl alcohol). Heliyon 2015, 1, e00038. [CrossRef]

32. Hamzeh, Y.; Sabbaghi, S.; Ashori, A.; Abdulkhani, A.; Soltani, F. Improving wet and dry strength properties of recycled old corrugated carton (OCC) pulp using various polymers. Carbohydr. Polym. 2013, 94, 577-583. [CrossRef] 